\title{
Synthesis of 1-[S-TAG-Substiturtedthioamido] Dicyandiamides
}

\author{
Dipak T. Tayade, Megha R. Raghuvanshi \& Rahul A. Bhagwatkar (Correponding author) \\ Department of Chemistry, S.R.R.L. Science College, MORSHI, Maharashtra 444905, India \\ Tel: 91-997-016-5107_E-mail: bhagwatkar83@gmail.com
}

Shashikant R. Aswale

Department of Chemistry, Lokmanya Tilak Mahavidyalaya, WANI, Maharashtra 445304, India

Received: September 20, 2010 Accepted: October 9, 2010 doi:10.5539/ijc.v3n2p74

\begin{abstract}
A novel series of 1-[S-TAG-N-substiturtedthioamido]dicyandiamides (5a-f) have been synthesized successfully by refluxing tetra-O-acetyl- $\beta$-D-glucopyraosylbromide (4) with cyanoamidinosubstitutedthiocarbamides (3a-f) in 2-propanol for $3 \mathrm{~h}$. The structure of these newly synthesized compounds have been established on the basis of chemical characteristics, elemental analysis, IR and ${ }^{1} \mathrm{H}-\mathrm{NMR}$ spectral analysis.
\end{abstract}

Keywords: $\quad$ S-Glucosylated, Tetra-O-acetyl- $\beta$-D-glucopyranosylbromide, dicyandiamides, cyanoamidinosubstituted-thiocarbamides

\section{Introduction}

Dicyandiamides showed noticeable pharmaceutical and biological values (Tayade D. T., 1997; Steinman, 1965). These heteroacycles were also cyclized in 5 and 6 membered heterocycles viz. thiadiazoles, dithiazoles, Hectors bases, thiadiazines and triazines. These heterocycles posses their own identity and significance in pharmaceutical, medicinal, agricultural, industrial and biotechnical sciences (Vora, 2009; El-Agrody, 2001; Hanessian, 2000; Rosowsky, A., 2004; Bamberger, 1983; Elvino Fako, 1951; Joshua, 1962; Deohate, 2005). S-glucosides and $\mathrm{N}$-glucosides had been found several applications in industry and also in medicinal chemistry (Irving Goodman, 1958; Deshmukh, 1986; Umar Ali, 1985).

An exhaustive literature survey about tetra-O-acetyl- $\beta$-D-glucopyranosylbromide (Lemieux, 1963) and tetra-O-benzoyl- $\beta$-D-glucopyranosylbromide showed that these two analog play the great role in the synthesis of S-glucosylated and N-glucosylated heteroacycles and heterocycles. Very few thioglucosides of thiocarbamide were reported earlier(Bedekar, 1981).

As evident from the structure of cyanoamidinosubstitutedthiocarbamide (Tayade, 1995 \& 2006), it was observed that there are various reactive sites in this molecule for the reactions. This molecule possesses $-\mathrm{SH},-\mathrm{CN},-\mathrm{NH}_{2}$ important reactive sites for the reactions. As a wider programme of this laboratory in the synthesis of nitrogen, nitrogen and sulphur containing heteroacycles and heterocycles. The interactions of dicyandiamide with various thioureas and alkyl/arylisothiocyanates had been investigated in sufficient details in various reaction conditions (Joshua, 1962; Tayade, 1997).

Herein we report our studies on the synthesis of 1-[S-TAG-N-substiturtedthioamido]dicyandiamides (5a-f) with the interactions of tetra-O-acetyl- $\beta$-D-glucopyranosylbromide (1) with cynoamidinosubstitutedthiocarbamides (3a-f) in 2-propanol medium were investigated to isolate title compound and the structure of these newly synthesized compounds have been established on the basis of chemical characteristics, elemental analysis and IR, ${ }^{1} \mathrm{H}-\mathrm{NMR}$ and mass spectral analysis.

\section{Materials and Methods}

\subsection{Cynoamidinosubmethylthiocarbamides (3d)}

Interaction of cyanoguanidine $(0.01 \mathrm{M})$ was carried out with methylisothiocyanate $(0.01 \mathrm{M})$ in acetone medium on water bath for 5 hours. It was filtered in hot condition. The resultant filtrate on distillation gave needle shaped yellowish crystals. The new product was dried at room temperature and recrystallized from aqueous ethanol and identified as cynoamidinosubstitutedthiocarbamides (3d), Yield $72 \%$ m.p. $181^{\circ} \mathrm{C}-183^{\circ} \mathrm{C}$. The reaction scheme 
was shown in Scheme-I. Similarly, other compounds (3a-f) were synthesised by above mentioned method and enlisted in Table-I.

\subsection{1-[S-TAG-N-phenylthioamido]dicyandiamides (5a)}

1-[S-TAG-N-phenylthioamido] dicyandiamide was synthesized by refluxing a mixture of tetra-O-acetyl- $\beta$-D-glucopyranosylbromide $(0.01 \mathrm{M})$ and cyanoamidinophenylthiocarbamide $(0.01 \mathrm{M})$ in 2-propanol medium, on water bath for 3 hrs. During refluxing the suspended tetra-O-acetyl- $\beta$-D-glucopyranosylbromide and cyanoamidinophenylthiocarbamide dissolve into the solution and clear solution was obtained. It was kept for $15 \mathrm{Hrs}$. at room condition. It was then mixed with distilled water, small quantity of semisolid was obtained, it was filtered [The separated aqueous solution was acidic to litmus and gave effervesces with sodium bicarbonate solution and was not desulphurized when boiled with alkaline plumbite solution.] The aqueous solution on basification with dilute ammonium hydroxide, the resultant filtrate on distillation gave needle shaped brown crystals. The new product was dried at room temperature and recrystallized from aqueous ethanol and identified as 1-[S-TAG-N-phenylthioamido]dicyandiamides (5a), yield$78 \%$, m. p. $167^{\circ}$ C. The reaction scheme was shown in Scheme-II. Similarly, other compounds $(5 \mathrm{~b}-\mathrm{f})$ were synthesised by above mentioned method and enlisted in Table-II.

\section{Instrumentation}

All the chemicals used were of Analar grade (India make). Alkyl/arylisothiocyanates were prepared according to literature method (Vogel, 1954), melting points of all synthesised compounds were determined in open capillary and uncorrected. IR-spectra were recorded on Perkin-Elmer spectrophotometer in the range $4000-400 \mathrm{~cm}^{-1}$ in $\mathrm{KBr}$ pellets. ${ }^{1} \mathrm{H}-\mathrm{NMR}$ spectra were recorded on Bruker AC-300F spectrometer with TMS as internal standard using $\mathrm{CDCl}_{3}$ and DMSO- $\mathrm{d}_{6}$. The purity of the compounds was checked on silica gel-G plates by TLC.

\section{References}

Bamberger, E. (1983). Ber. dtsch. Chem. Ges., 16, 1460.

Bedekar, I. D., Paranjape, M. G. (1981). J. Indian Chem. Soc., 58, 1014.

Deohate, P.P., Berad, B.N. (2005). Indian J. Chem., 44B, 638-642.

Deshmukh, S. P., Berad, B. N., Paranjpe, M.G. (1986). J. Indian Chem. Soc., 63, 315.

El-Agrody, A. M., Abd El-Latif, El-Hady, M. S. (2001). Molecules, 6, 519-527.

Elvino Fako, Shirley. D., George Hitching. (1951). J. Am. Chem. Soc., 73, 3760-3766.

Hanessian, S., Sgarbi, P. W. M. (2000). Bioorg. Med. Chem. Lett., 10, 437-443.

Irving Goodman. (1958). Advances in Carbonhydrate Chemistry, 13, 233, Academic Pres, INC, Publisher, New York.

Joshua, C. P. (1962). Chemistry of Hector's Base, Ph. D., Thesis, B.H.U.

Joshua, C. P. (1962). J. Sci. Indus. Res., 21B, 688-690.

Lemieux, R. U. (1963). Methods Carbohydr. Chem., 2, 221.

Rosowsky, A., Queener, S. F. (2004). J Med Chem., 47, 1475.

Steinman, G., Lemmon, R. M., Melvin Calvin. (1965). Science, 147, 3665, 1574 - 1575.

Tayade D. T. (1997). Oriental,J. Chem., 13(2), 309-310.

Tayade, D. T. (1997). Oriental J. Chem., 13(2), 189-190.

Tayade, D. T., Chincholkar, M. M. (1995). Acta Ciencia Indica, XXI(1), 37-38.

Tayade, D. T., Waghmare, J. S., Patil, S. U. (2006). J. Indian Chem. Soc., 83, 1-3.

Umar Ali, M., Meshram, H. M., Paranjpe, M. G. (1985). J. Indian Chem. SoC., 62, 666.

Vogel, A. Z. (1954). Text book of Practical Organic Chemistry including Qualitative Organic analysis, ELBS Landon Greek and Co., Ltd, ISBN 058244245 1, 615.

Vora, J. J., Vasava, S. B., Patel, A. D., Parmar, K. C., Chauhan, S. K. Sharma, S. S. (2009). E-Journal of Chemistry, 6(1), 201-206. 


\section{Reaction Schemes}

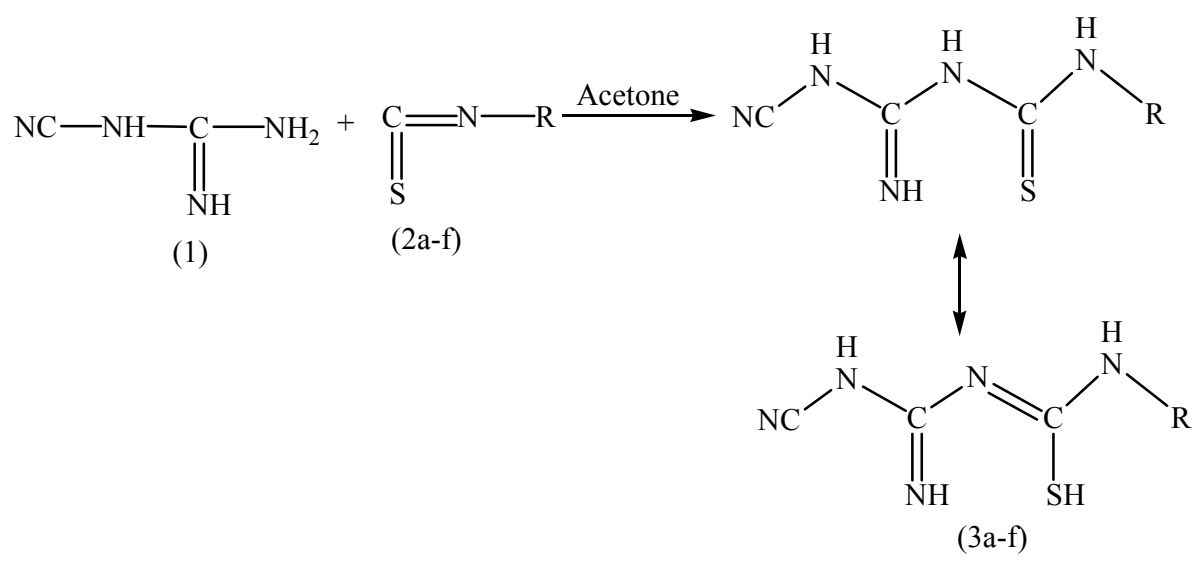

Scheme-I

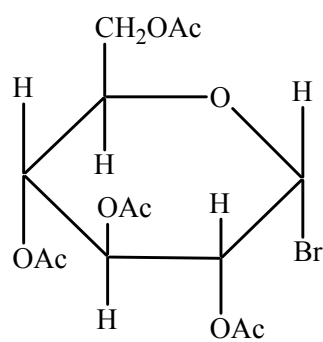

(4)
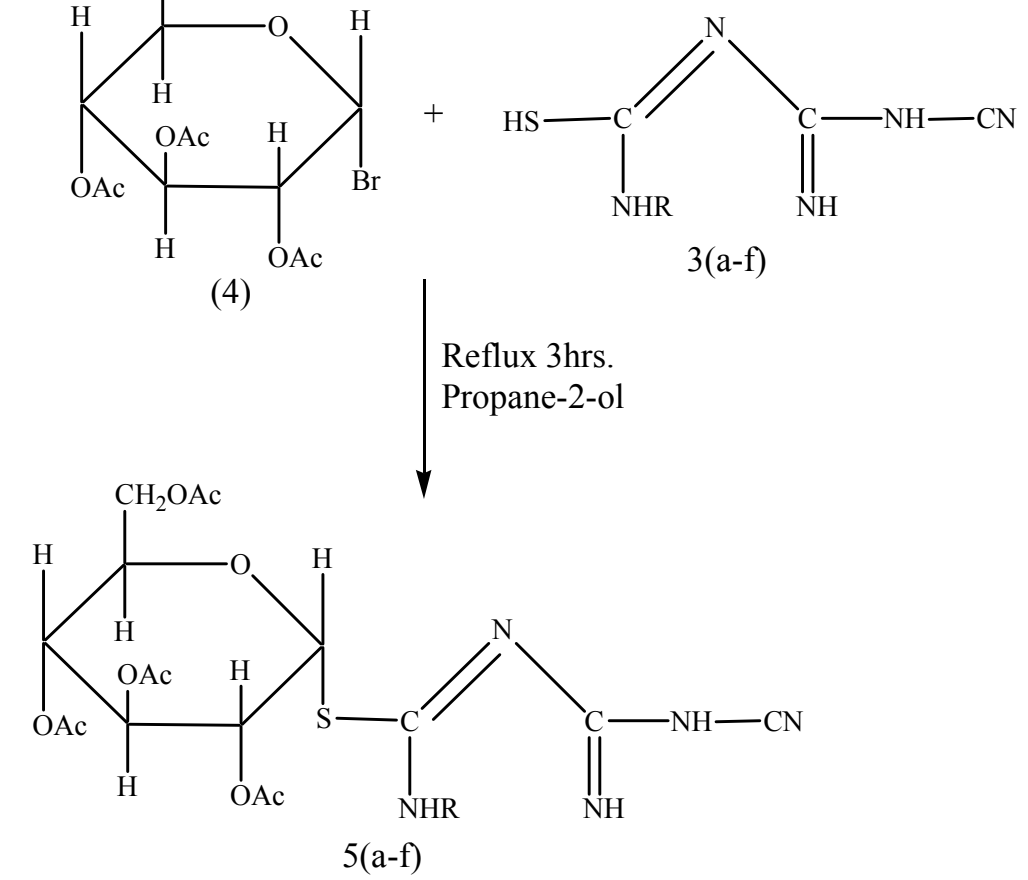

Scheme-II

\section{Physical data for synthesised compounds}


Table 1.

\begin{tabular}{|l|l|l|l|}
\hline Compound No. & Cyanoamidinosubstitutedthiocarbamide & Yield $(\%)$ & $\mathrm{m} . \mathrm{p} .\left({ }^{0} \mathrm{C}\right)$ \\
\hline $3 \mathrm{a}$ & -phenyl- & 72 & 188 \\
\hline $3 \mathrm{~b}$ & -p-Cl-phenyl- & 67 & 149 \\
\hline $3 \mathrm{c}$ & -p-tolyl- & 63 & 168 \\
\hline $3 \mathrm{~d}$ & - methyl & 72 & 182 \\
\hline $3 \mathrm{e}$ & -ethyl- & 71 & 189 \\
\hline $3 \mathrm{f}$ & -t-butyl- & 69 & 180 \\
\hline
\end{tabular}

Table 2.

\begin{tabular}{|c|c|c|c|}
\hline Compound No. & 1-[S-TAG-N-substitutedthioamido]dicyandiamide & Yield \% & m.p. $\left({ }^{0} \mathrm{C}\right)$ \\
\hline $5 a$ & -phenyl- & 78 & 167 \\
\hline $5 b$ & -p-Cl-phenyl- & 61 & 164 \\
\hline $5 \mathrm{c}$ & -p-tolyl- & 65 & 148 \\
\hline $5 \mathrm{~d}$ & -methyl- & 68 & 161 \\
\hline $5 e$ & -ethyl- & 64 & 159 \\
\hline $5 \mathrm{f}$ & -t-butyl- & 63 & 142 \\
\hline
\end{tabular}

Table 3. Chemical data of synthesized compounds for (3a-f)

\begin{tabular}{|l|l|l|l|l|l|l|l|l|l|l|l|l|}
\hline & 3a & \multicolumn{3}{l}{ 3b } & \multicolumn{3}{l|}{ 3c } & \multicolumn{3}{l|}{ 3d } & \multicolumn{3}{l|}{ 3f } \\
\hline & Calcd. & Found. & Calcd. & Found. & Calcd. & Found. & Calcd. & Found. & Calcd. & Found. & Calcd. & Found. \\
\hline $\mathrm{C}$ & 49.32 & 49.28 & 42.60 & 42.58 & 51.50 & 51.40 & 30.57 & 30.44 & 35.09 & 35.03 & 42.21 & 42.20 \\
\hline $\mathrm{H}$ & 4.11 & 4.09 & 3.16 & 3.21 & 4.72 & 4.70 & 4.46 & 4.34 & 5.26 & 5.21 & 6.53 & 6.50 \\
\hline $\mathrm{N}$ & 31.96 & 31.83 & 27.61 & 27.60 & 30.04 & 30.12 & 44.59 & 44.63 & 40.93 & 41.03 & 35.18 & 35.55 \\
\hline $\mathrm{S}$ & 14.61 & 14.52 & 12.62 & 12.62 & 13.73 & 13.66 & 20.38 & 20.20 & 18.71 & 18.33 & 16.08 & 15.73 \\
\hline $\mathrm{Cl}$ & & & 14.00 & 13.86 & & & & & & & & \\
\hline
\end{tabular}

Table 4. Chemical data of synthesized compounds for (5a-f)

\begin{tabular}{|l|l|l|l|l|l|l|l|l|l|l|l|l|}
\hline & \multicolumn{3}{|l}{$5 \mathrm{a}$} & \multicolumn{3}{l}{$5 \mathrm{~b}$} & \multicolumn{2}{l}{$5 \mathrm{c}$} & \multicolumn{2}{l|}{$5 \mathrm{~d}$} & \multicolumn{3}{l|}{ 5f } \\
\hline & Calcd. & Found. & Calcd. & Found. & Calcd. & Found. & Calcd. & Found. & Calcd. & Found. & Calcd. & Found. \\
\hline $\mathrm{C}$ & 50.27 & 49.98 & 47.30 & 46.70 & 51.24 & 51.20 & 44.35 & 43.12 & 45.51 & 45.63 & 47.63 & 46.32 \\
\hline $\mathrm{H}$ & 4.92 & 4.80 & 4.46 & 4.50 & 4.98 & 4.90 & 5.13 & 4.97 & 5.39 & 5.12 & 5.86 & 5.43 \\
\hline $\mathrm{N}$ & 12.75 & 12.22 & 11.99 & 11.89 & 12.46 & 12.00 & 14.37 & 14.34 & 13.97 & 13.23 & 13.23 & 12.44 \\
\hline $\mathrm{S}$ & 5.83 & 5.80 & 5.48 & 5.33 & 5.69 & 5.09 & 6.57 & 6.44 & 6.39 & 6.21 & 6.05 & 5.09 \\
\hline $\mathrm{Cl}$ & & & 6.08 & 6.00 & & & & & & & & \\
\hline
\end{tabular}


Table 5. IR spectra of synthesised compounds in $\left(\mathrm{cm}^{-1}\right)$

\begin{tabular}{|l|l|l|l|l|l|l|l|}
\hline Compound & $v(\mathrm{~N}-\mathrm{H})$ & $v$ Ar-H & $v(>\mathrm{C}=\mathrm{NH})$ & $v(>\mathrm{C} \equiv \mathrm{N})$ & $v(>\mathrm{C}-\mathrm{N})$ & $v \mathrm{Ph}-\mathrm{Cl}$ & $v(>\mathrm{C}-\mathrm{S})$ \\
\hline$(3 \mathrm{a})$ & 3268.6 & 3057.3 & 1653.6 & 2350.5 & 1422.4 & --- & 737.0 \\
\hline$(3 \mathrm{~b})$ & 3274.2 & 3100.9 & 1629.6 & 2411.5 & 1375.7 & 842.6 & 742.3 \\
\hline$(3 \mathrm{c})$ & 3304.9 & 3049.2 & 1642.5 & 2321.9 & 1365.8 & --- & 742.6 \\
\hline$(3 \mathrm{~d})$ & $3195.5,3082.1$ & --- & 1680.1 & 2396.2 & 1390.2 & & 721.8 \\
\hline$(3 \mathrm{e})$ & $3233.1,3176.2$ & --- & 1672.4 & 2341.7 & 1333.4 & --- & 733.7 \\
\hline$(3 \mathrm{f})$ & $3219.8,3159.3$ & --- & 1644.2 & 2383.5 & 1310.5 & --- & 790.3 \\
\hline$(5 \mathrm{a})$ & 3184.8 & 2931.3 & 1533.8, & 2352.7 & 1324.5 & --- & 746.2 \\
\hline$(5 \mathrm{~b})$ & 3290.4 & 3044.6 & 1653.6 & 2353.5 & 1321.6 & 810.3 & 740.2 \\
\hline$(5 \mathrm{c})$ & 3241.3 & 3062.5 & 1642.7 & 2375.4 & 1379.5 & --- & 713.5 \\
\hline$(5 \mathrm{~d})$ & $3249.6,3184.3$ & --- & 1690.4 & 2378.3 & 1352.6 & & 711.3 \\
\hline (5e) & $3255.3,3122.7$ & --- & 1623.4 & 2344.5 & 1329.4 & --- & 741.5 \\
\hline (5f) & $3216.3,3119.8$ & --- & 1611.8 & 2367.9 & 1309.4 & --- & 734.2 \\
\hline
\end{tabular}

Table 6. NMR spectra of synthesised compounds in (ppm)

\begin{tabular}{|l|l|l|l|l|l|}
\hline Compound & Ar-NH & Ar-H & N-H & $\mathrm{CO}-\mathrm{CH}_{3}$ & $\mathrm{C}-\mathrm{H}$ \\
\hline$(3 \mathrm{a})$ & $\delta 8.4-7.5$ & $\delta 5.8-6.4$ & $\delta 3.9-4.5$ & --- & --- \\
\hline$(3 \mathrm{~b})$ & $\delta 9.4-8.1$ & $\delta 6.4-7.1$ & $\delta 3.2-3.8$ & --- & --- \\
\hline$(3 \mathrm{c})$ & $\delta 8.6-7.7$ & $\delta 6.3-7.1$ & $\delta 4.0-4.5$ & --- & $\delta 1.0-1.4$ \\
\hline$(3 \mathrm{~d})$ & --- & --- & $\delta 3.7-4.9$ & --- & $\delta 0.9$ \\
\hline$(3 \mathrm{e})$ & --- & --- & $\delta 4.5-4.7$ & --- & $\delta 1.2-1.5$ \\
\hline$(3 \mathrm{f})$ & --- & --- & $\delta 3.9-4.4$ & --- & $\delta 3.97$ \\
\hline$(5 \mathrm{a})$ & $\delta 8.9-8.1$ & $\delta 5.8-6.4$ & $\delta 4.5-4.8$ & $\delta 2.5-3.0$ & \\
\hline$(5 \mathrm{~b})$ & $\delta 9.0-8.5$ & $\delta 6.1-6.5$ & $\delta 4.3-4.9$ & $\delta 2.1-2.5$ & \\
\hline$(5 \mathrm{c})$ & $\delta 8.7-7.8$ & $\delta 6.5-7.0$ & $\delta 4.2-4.4$ & $\delta 2.4-2.6$ & $\delta 1.2-1.5$ \\
\hline$(5 \mathrm{~d})$ & --- & --- & $\delta 4.0-4.6$ & $\delta 2.6-3.7$ & $\delta 1.4$ \\
\hline$(5 \mathrm{e})$ & --- & --- & $\delta 4.1-4.3$ & $\delta 2.4-2.8$ & $\delta 1.3-1.7$ \\
\hline$(5 \mathrm{f})$ & --- & --- & $\delta 3.8-4.2$ & $\delta 2.5-3.5$ & $\delta 4.6$ \\
\hline
\end{tabular}

